\title{
Entrelacs
}

Cinéma et audiovisuel

Hors-série $n^{\circ} 4$ | 2016

Paysages en séries

\section{L'océan comme cadre narratif : Une analyse des différentes perceptions du paysage marin dans The Affair}

L'océan comme cadre narratif:

\section{Sandrine Chapon}

\section{(2) OpenEdition}

Journals

Édition électronique

URL : http://journals.openedition.org/entrelacs/2146

DOI : $10.4000 /$ entrelacs.2146

ISBN : 2261 - 5482

ISSN : 2261-5482

Éditeur

Éditions Téraèdre

Édition imprimée

Date de publication : 1 novembre 2016

ISSN : 1266-7188

\section{Référence électronique}

Sandrine Chapon, «L'océan comme cadre narratif : Une analyse des différentes perceptions du paysage marin dans The Affair », Entrelacs [En ligne], Hors-série n 4 | 2016, mis en ligne le 06 février 2018, consulté le 20 avril 2019. URL : http://journals.openedition.org/entrelacs/2146 ; DOI : 10.4000/ entrelacs. 2146

Ce document a été généré automatiquement le 20 avril 2019

Tous droits réservés 


\title{
L'océan comme cadre narratif : Une analyse des différentes perceptions du paysage marin dans The Affair
}

\author{
L'océan comme cadre narratif:
}

\section{Sandrine Chapon}

1 The Affair, diffusée par la chaîne Showtime depuis 2014, a reçu le Golden Globe de la meilleure série dramatique en 2015. Comme l'évoque son titre, la série américaine explore les méandres émotionnels d'une relation extra-conjugale entre Alison Lockhart, une serveuse native de Montauk - une petite ville balnéaire de Long Island - et Noah Solloway, un professeur de littérature New-Yorkais qu'elle rencontre lors des vacances familiales de ce dernier. L'histoire d'amour est mêlée à une enquête judiciaire dont ne nous ne percevons que des fragments au cours des deux premières saisons. La relation qui naît entre les personnages est révélée par le biais d'une structure narrative complexe qui interroge la subjectivité des relations humaines. La première moitié de chaque épisode est narrée selon le point de vue d'un des personnages. Cette " vérité ", construite par le premier protagoniste, est complétée et modifiée par la version de son interlocuteur dans la seconde moitié de l'épisode. Le double point de vue narratif est, par ailleurs, encore déconstruit par une voix-off, celle des personnages qui répondent aux questions de la police, donnant une version des faits encore différente de ce qui nous est montré à l'image. Enfin, Noah, qui est aussi écrivain, puise dans cette relation extra-conjugale matière à alimenter la diégèse d'un roman qu'il est en train d'écrire, constituant une quatrième version des faits.

Dans une interview accordée à la chaîne Showtime en 2015, Sarah Treem et Hagai Levi les créateurs de The Affair - expliquent que Montauk est le personnage principal de la série et constitue le cadre servant à faire progresser l'histoire ${ }^{1}$. En effet, de très nombreuses scènes sont tournées en extérieur et des conversations récurrentes ont pour objet la situation géographique, économique et esthétique du littoral. $\mathrm{Si}$, comme l'affirment les créateurs, le paysage marin est un protagoniste à part entière, comment les personnages physiques de cette fiction entrent-ils en relation avec lui? C'est la 
question que nous proposons d'explorer dans cet article. Nous verrons, à travers une lecture analytique des deux premières saisons que, tout comme les relations humaines de la série, le paysage marin est interprété de manière subjective en fonction des liens que les personnages tissent avec ce littoral. Nous montrerons, dans une première partie, que Montauk est l'objet de tensions fortes entre ceux qui voient le paysage comme un cadre de vie et ceux qui le perçoivent comme une ressource économique. Dans un deuxième temps nous verrons que le paysage marin est pour les deux protagonistes l'objet de projection de leurs émotions.

\section{Le paysage, approche sociale et économique}

\section{Le paysage ressource}

3 Montauk est un petit village de pêcheur situé à l'extrémité orientale de la péninsule de Long Island dans l'État de New York. Ce hameau de quelque trois mille habitants fait partie de la région des Hamptons vers lequel convergent tous les étés les New-Yorkais les plus riches. Échappant à la chaleur suffocante de Manhattan, ces citadins huppés viennent renouer avec l'atmosphère feutrée de Gatsby le Magnifique ${ }^{2}$ ou des paysages ruraux peints par Edward Hopper. Tout comme les tableaux de ce peintre, la série témoigne des ambivalences de la société de la Nouvelle-Angleterre en brossant les oppositions entre la nature et la cité. Montauk est en effet peuplée par deux catégories d'individus qui entretiennent des rapports très différents avec le paysage.

D'un côté se trouvent ceux qui sont prêts à sacrifier le paysage sur l'autel de son attrait économique en développant des projets urbains au risque de dénaturer l'identité culturelle de la région. À l'autre bout du spectre se trouvent ceux qui se posent en gardiens du paysage en tant que bien public à préserver. La figure du défenseur de la nature est incarnée par le personnage de Cole, le mari d'Alison, qui, quand il n'est pas en train de surfer ou faire des feux de camp sur la plage à la nuit tombée, dédie son énergie à la préservation du littoral atlantique.

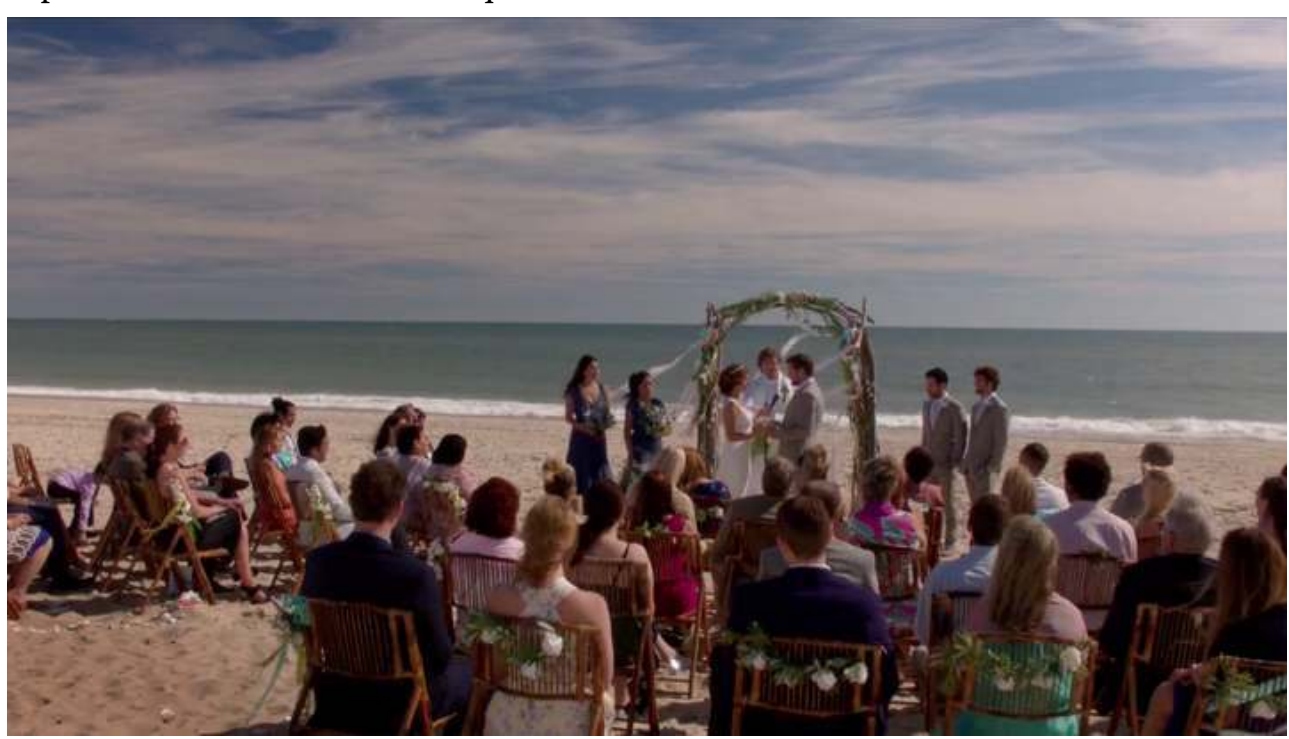

Fig 1 
5 Très attaché à son cadre de vie (au point d'avoir fait le choix de se marier sur la plage), il se bat contre les diverses tentatives d'aménagement du territoire qui ont pour fonction d'attirer plus de touristes, mais il cristallise par là-même la colère des locaux qui voient dans le paysage marin une opportunité économique. Les passions qu'éveillent ce sujet illustrent que, bien plus qu'un simple milieu physique, le paysage relève de la représentation mentale car il symbolise, pour les uns, la mémoire collective des habitants de Montauk et une source de plaisir esthétique alors qu'il n'est pour les autres qu'un bien de consommation. La série ironise en quelque sorte sur le fait que, hormis Cole le mari d'Alison, les personnages qui veulent rentabiliser le paysage sont plus particulièrement les locaux et ceux qui veulent le préserver, les touristes.

6 Les émotions procurées par la beauté du littoral sont aussi ressenties par le spectateur grâce à de longs plans fixes de paysages marins dans lesquels figurent peu de personnages. Ces cadrages établissent un écho à la "scène américaine », un style de peinture issu du mouvement naturaliste qui est apparu pendant la première moitié du XIXème siècle aux États-Unis, en opposition à l'art moderne : ce sont des artistes comme Edward Hopper qui initient ce mouvement pictural nouveau, gage d'une certaine fierté à l'égard des paysages vierges de la côte Est. Les auteurs de scènes américaines s'attachent particulièrement au traitement de la lumière, conférant à leurs toiles sérénité et clarté. La série reprend cette dialectique et illustre le mode de vie des néo-hippies vivant à Montauk à l'année, loin de l'agitation de la ville qui n'est qu'à quelques heures en train. L'ambiance de bout du monde (The End est le nom donné à la pointe de la péninsule) établit un contraste visuel saisissant avec la mégalopole et de nombreux dialogues portent sur le statut de la ville par rapport à celui de la nature, à l'instar de cette réplique relative à l'odeur qui règne à Brooklyn :

7 Cole: Ça sent l'enfer ici. [...] tu sens ça ? Il faut vraiment être suicidaire pour vouloir vivre ici. [...] J'ai l'impression que je vais vomir. ${ }^{3}$

8 L'idéal de retour à la nature porté par Cole n'est pas pour autant exempt de contradictions puisque l'on apprend assez rapidement dans la première saison que ce dernier est à la tête d'un trafic de cocaïne à destination des touristes venus se ressourcer sans tout à fait se départir de leurs habitudes toxiques. La contradiction dans la représentation des valeurs véhiculées par le paysage n'est pourtant pas l'apanage des natifs de Montauk. Les «summer people», comme les appellent les locaux non sans un certain mépris, sont aussi au centre de conflits de valeurs.

\section{Le paysage " signe extérieur de richesse »}

9 Si la plupart des scènes de la première saison ont lieu à Montauk et sont tournées en extérieur, la parenthèse des vacances finit néanmoins par se refermer et les New-Yorkais retournent à la ville à la fin de l'été. La série, particulièrement la deuxième saison, propose donc aussi de nombreuses scènes d'intérieur. Il est intéressant de noter qu'une grande quantité d'aquarelles marines ornent les murs des maisons des citadins de la série.

10 Ces peintures possèdent en premier lieu une fonction décorative en offrant une respiration au cœur de la ville qui est présentée comme une mégalopole tentaculaire, suffocante et toujours en mouvement. Par ailleurs, les marines sont aussi un signe extérieur de richesse car elles rappellent aux visiteurs que ces New-Yorkais sont suffisamment aisés pour posséder une résidence secondaire dans les Hamptons, l'un des 
lieux de villégiature les plus prisés de la côte Est. En effet, si l'on applique la théorie de la consommation ostentatoire développée par le sociologue et économiste américain Thorstein Veblen (1857-1929) à la série, ces New-Yorkais possèdent des tableaux illustrant l'imagier des Hamptons dans l'objectif plus ou moins conscient de montrer leur appartenance à une classe sociale élevée. Le paysage marin est donc instrumentalisé afin de devenir un marqueur social.

11 S'ils affichent de manière visible qu'ils passent leurs vacances à la mer, les estivants n'investissent pas pour autant le paysage en tant qu'espace de vie. Toutes les résidences secondaires de la série possèdent en effet une « vue sur la mer » mais le paysage demeure pour eux une sorte de toile de fond, un élément de décor à regarder depuis la terrasse en sirotant un verre de vin (fig.2).

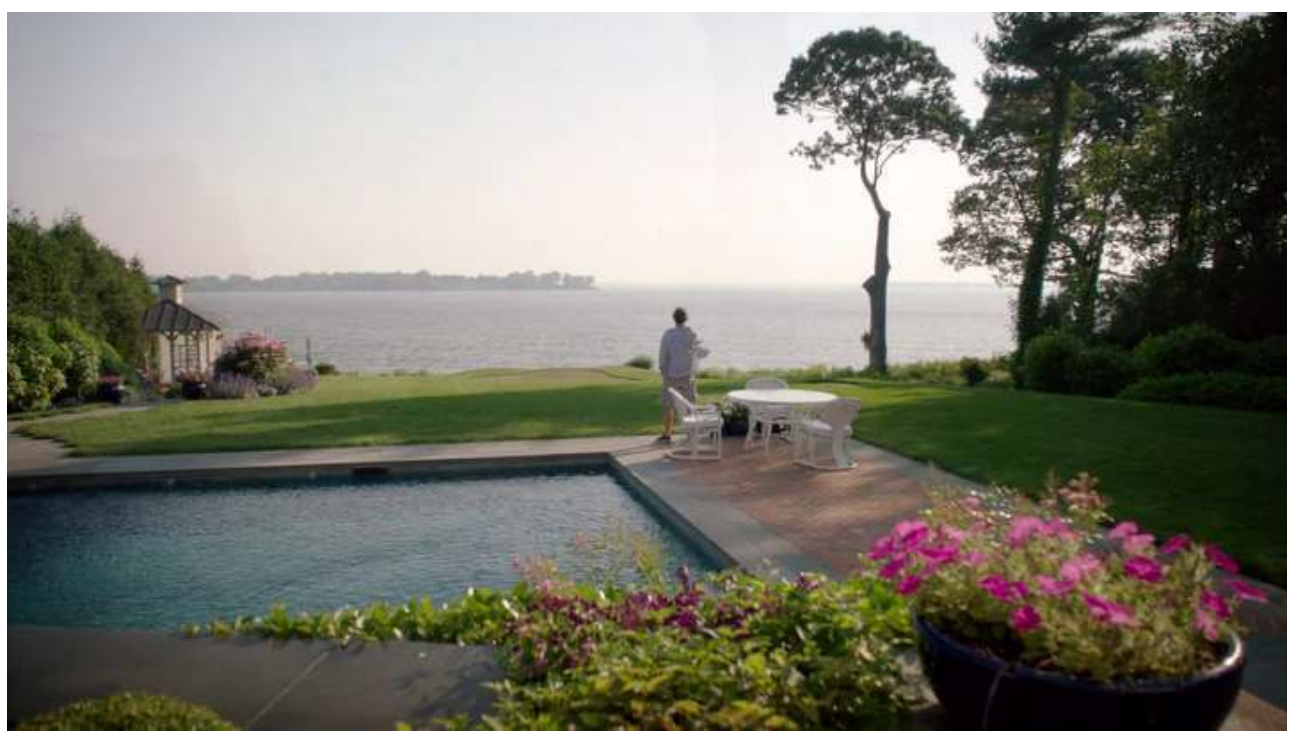

Fig. 2

Contrairement aux habitants de Montauk, aucun des vacanciers ne semble se baigner dans la mer, à laquelle ils préfèrent leur piscine privée. Même si le protagoniste principal précise que l'eau de la piscine est salée, elle ne présente pas les inconvénients de la plage tels que le sable, les rouleaux ou encore les algues. Le lieu de villégiature représente donc un pseudo-retour à la nature qui se fait de manière aseptisée, dépouillée des inconforts inhérents à son aspect sauvage. La forte fréquentation d'une boite de nuit qui vient de se construire en bord de mer est un détail supplémentaire visant à illustrer ce paradoxe de citadins ne fuyant leur milieu que pour y reproduire les mœurs de la cité. En effet, malgré le nom de la boite de nuit, The End, c'est bien pour s'adonner à la drogue et au bruit qu'ils fréquentent ce lieu pourtant prometteur de silence et de pureté.

Cette fonction instrumentale du paysage est violemment rejetée par Witney, la fille aînée des Solloway. Adolescente écorchée, elle a bien compris que les aquarelles marines et les maisons secondaires avec vue sur la mer sont de jolies constructions dont la seule fonction est de masquer une réalité bien moins reluisante. C'est pour cette raison que, de manière très symbolique, elle décroche les tableaux des murs de sa chambre quand elle arrive à Montauk, comme pour protester contre le vernis social symbolisé par les aquarelles marines. En effet, celui-ci craquelle assez rapidement dans la première saison malgré l'entêtement de Noah à nier qu'il n'est pas heureux. C'est d'ailleurs ce dernier qui remet les tableaux au mur lors de leur départ précipité de Montauk et suite à la 
révélation de sa liaison extra-conjugale, comme pour tenter de maintenir à tout prix l'illusion que l'équilibre de la famille n'a pas été bouleversé par la trahison du père.

\section{Le paysage extérieur comme psycho-système}

14 La série permet d'illustrer le concept de paysage en tant que "psycho-système ", selon le terme forgé par le géographe Pierre Arnould ${ }^{4}$ en 2003, c'est-à-dire un lieu de projections inconscientes dans lequel l'imaginaire, les émotions, les mythes ou encore les symboles colorent la perception du paysage. Nous verrons que les personnages lisent ce dernier en fonction de leurs fantasmes respectifs.

\section{Paysage et projection érotique}

Noah Solloway enseigne la littérature dans un lycée public de New York et vient de publier un premier roman qui rencontre un succès pour le moins modéré. Il est marié à une riche New-Yorkaise et est père de quatre enfants. Les vacances estivales arrivent et, comme tous les ans, les Solloway quittent leur maison de Brooklyn pour aller passer l'été dans la villa des beaux-parents de Noah. La première scène du pilote révèle que ce personnage est doté d'un surmoi puissant : il tâche d'ignorer en permanence les indices de sa propre insatisfaction quant à la vie qu'il mène. Quand il est interrogé par la police sur son état d'esprit à l'époque de sa rencontre avec Alison, c'est plus pour se persuader lui-même que pour convaincre l'officier qu'il affirme qu'il était heureux et fier de sa famille ${ }^{5}$. Malgré le fantasme d'homme épanoui dont il essaie de se convaincre, il nous est montré comme un homme dont la fonction de père a largement pris l'ascendant sur son rôle de mari. Sa vie sexuelle est systématiquement frustrée par l'interruption d'un de ses enfants dans le lit conjugal et ses deux ainés sont des adolescents rebelles qui rendent les moments en famille désagréables. Ce n'est pas non plus grâce à son salaire qu'il peut trouver matière à affirmer sa virilité dans son foyer: ce sont ses beaux-parents qui financent leur train de vie en leur ayant acheté une maison de Brooklyn, en payant l'école privée des enfants ou encore en les accueillant dans leur résidence secondaire tous les étés. Cette situation de dépendance financière entraîne une relation malsaine. En effet, la belle-mère, investie d'un pouvoir sur le couple grâce à l'argent qu'elle lui donne, interfère dans ses choix éducatifs et occupe une fonction centrale, particulièrement castratrice, qui conduit à des tensions au sein de la famille. Par ailleurs, les beaux-parents de Noah ne cachent pas qu'ils n'ont jamais cautionné le mariage de leur fille. Bruce Butler, écrivain célèbre de littérature populaire, méprise souverainement son gendre et ne manque pas une occasion de lui signifier sa désapprobation. Enfin, Noah subit une importante pression sociale relative à sa capacité à écrire un second roman car, comme l'exprime avec dédain son beau-père, « un premier roman est à la portée de tout le monde mais presque personne n'est capable d'en écrire un second $»^{6}$.

16 C'est dans ce contexte suffocant que nait la liaison entre Alison et Noah. Celle-ci n'aurait pu exister ailleurs que dans les espaces ouverts du littoral. Puisqu'aucun des lieux clos de la vie de Noah ne lui appartient vraiment, il est obligé de fuir vers l'extérieur pour échapper à l'univers castrateur dans lequel il est enfermé. C'est donc sur la plage, le long de la côte sauvage qu'il va se réapproprier sa vie, sa sexualité et sa force créatrice. On le voit par conséquent faire des footings dans la campagne ou se promener la nuit sur la plage. C'est là que commenceront à s'exprimer ses désirs pour Alison, la fille de Montauk. 

très forte charge sexuelle. L'intérieur symbolisant la contrainte et la frustration, l'extérieur devient le lieu de l'épanouissement des sens. Il est intéressant de noter à ce propos que, lors d'une soirée organisée chez les beaux-parents, Alison et Noah tentent de s'offrir un moment d'intimité en s'échappant sur la plage privée de la résidence à laquelle on accède par une porte munie d'un code. La porte leur résiste car Noah a oublié le code mais finit par se souvenir qu'il correspond à la date d'anniversaire de sa femme, rappel subtil que l'épouse de Noah est un obstacle à la relation illégitime qui va avoir lieu de l'autre côté de la barrière.

L'attirance que développe Noah à l'égard d'Alison colore sa perception du littoral et de ses habitants; aussi s'opère-t-il dans l'esprit de ce dernier un amalgame entre paysage, liberté et sentiment amoureux. Dans les scènes tournées selon le point de vue de Noah, Alison est toujours vêtue de petites robes d'été qui sont soulevées par le vent alors que dans les souvenirs de celle-ci, elle se protège du froid en couvrant ses épaules de vieilles couvertures ou en portant des jeans.

Les projections romantiques de Noah font d'ailleurs l'objet d'une conversation avec sa maitresse, lorsqu'il manifeste l'envie de partir en mer avec un pêcheur pour effectuer des repérages pour son second livre:

Noah : Tu ne m'en crois pas capable?

Alison: Je pense que tu es un estivant.

Noah: Qu'est-ce que tu entends par là?

Alison : Je pense que ta représentation de la vie d'ici est un fantasme. C'est une petite ville idyllique. Nous sommes des gens simples, humbles. Nous aimons la mer, n'est-ce pas?

Noah : Et donc c'est comment en vrai ?

La réponse que formule Alison permet de voir qu'elle perçoit le paysage marin à travers un filtre radicalement différent car, comme elle lui précise, « c'est dur tu sais. Tu pars la nuit, tu ne reviens pas avant le petit matin. Tu dors par roulement. Il fait froid et humide. L'océan est mauvais ${ }^{8}$.

\section{Paysage et projection mortifère}

Très tôt dans la première saison, Alison se fait le guide de Noah dans la presqu'île car il a besoin de s'imprégner de l'ambiance de la vie à Montauk pour étayer son roman. Les balades sur la côte sauvage servent aussi de prétexte aux personnages pour vivre leur liaison. Alison fait découvrir son univers intérieur en racontant le paysage. Ces récits permettent de percevoir le rapport très ambivalent qu'elle entretient avec l'élément marin.

Pour ce personnage, la mer a été désacralisée par l'industrie de la pêche qui a détruit l'écosystème, une action qu'elle qualifie de «viol » de l'océan. Cette mer, qui employait 8000 personnes par le passé, permet tout juste de subvenir aux besoins de quelque 200 pécheurs à l'heure actuelle. Pour Alison, la mer, qui nourrissait les habitants de Montauk, est maintenant selon ses propres mots « stérile » et « morte ».

Au-delà de la lecture économique du paysage, l'océan est une métaphore de la personnalité complexe d'Alison. Lors de leurs pérégrinations, elle emmène Noah sur l'île de Block pour lui faire découvrir son paysage préféré, la falaise des Mohegan bluffs, ainsi 
nommée parce qu'elle fut au XVI ${ }^{\text {ime }}$ siècle le siège d'une bataille particulièrement meurtrière dans laquelle les amérindiens Niantic ont refoulé les Mohegan qui tentaient de les envahir, en les poussant au-dessus de la falaise. Dans un récit emprunt de nostalgie ( "J'étais une fan d'épaves quand j'étais enfant ${ }^{9}$ ", précise-t-elle au quatrième épisode), elle décrit sa fascination pour ces nombreuses tragédies. Les paysages de Montauk ont, en effet, été le lieu d'épisodes meurtrier mais aussi de naufrages de navires. Selon les dires d'Alison, par jour de grand vent, on peut y entendre la voix d'un petit garçon appeler sa maman qui s'est noyée.

Les cris de cet enfant et l'attirance d'Alison pour ces voix fantômes font écho à sa blessure personnelle car on apprend, au bout de quelques épisodes, que son propre fils de quatre ans s'est noyé en mer. Pour Alison, la mer est donc essentiellement un lieu qui donne la mort. Cette information permet mieux de comprendre pourquoi, dans le premier épisode, elle enjoint son mari de faire attention quand il part surfer alors que la mer est belle et semble calme. Quelques épisodes plus tard, dans une scène tournée sur la plage et qui joue sur l'absence de focalisation, elle explique en voix-off au policier qui l'interroge, que ses souvenirs de cette époque sont flous en raison de la peine d'avoir perdu son fils. Elle raconte aimer précisément cet endroit parce que les vagues y donnent l'impression d'être encore plus en colère contre la vie qu'elle.

Ces informations donnent aussi des clés pour comprendre le générique de la série. En effet, si l'affiche de la série - dans laquelle on voit Noah et Alison presque complètement immergés dans une eau bleue et paisible - est une sorte de promesse de scènes sensuelles, le générique de la série en donne une impression opposée.

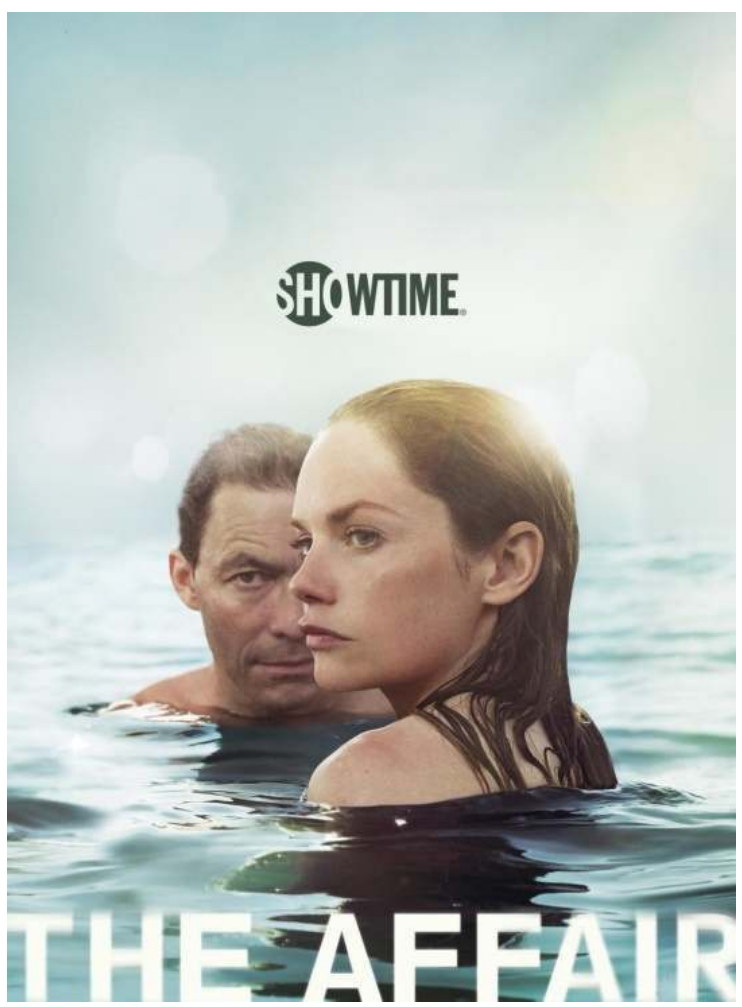

Fig 3 


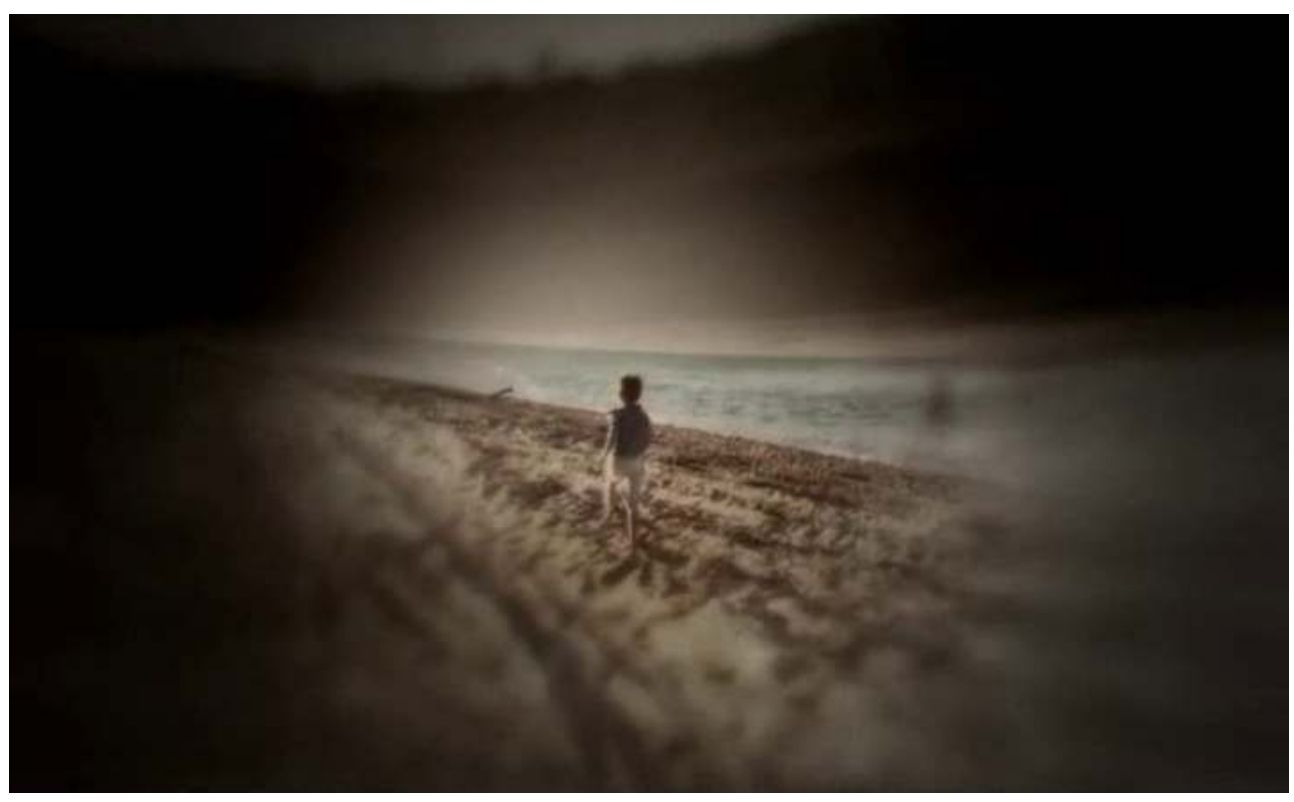

Fig 4

31 Le générique d'ouverture est cadré essentiellement sur l'élément marin mais c'est un océan déchaîné, noir et menaçant qui est filmé, le tout en caméra subjective. Ses vagues submergent les personnages et semblent engloutir tout ce qui a le malheur de s'en approcher. Le seul plan en couleur est celui dans lequel un enfant court sur la plage, or d'une part la couleur est surannée - ce qui donne l'impression que la scène appartient au passé - et d'autre part l'enfant s'éloigne de la caméra et n'est visible que de dos. La profondeur de champ est extrêmement courte, une grande partie de l'image est floue, ce qui donne l'impression d'un souvenir qui s'efface imperceptiblement.

La mer déchaînée, "mauvaise ", est ainsi une projection du paysage intérieur d'Alison, tourmentée et autodestructrice. Elle nous apparaît comme rongée par la culpabilité d'avoir causé la mort de son fils et d'avoir précipité la destruction de son mariage. $\mathrm{Si}$ Alison redoute la mer (elle dit ne même pas savoir nager) elle est pourtant indissociable de l'élément marin. Elle se présente comme la fille du vent (« ma mère, où est-elle ? je ne sais pas vraiment. Elle est comme le vent, elle va où elle veut. Elle fait ce qu'elle veut $\left.{ }^{10} »\right)$ et s'identifie à l'océan. Si Noah est attiré par le côté sauvage de sa maîtresse, il commet l'erreur de penser qu'il peut l'arracher à son milieu et la transposer dans son monde. Après lui avoir assuré qu'ils vivront à Montauk quand il aura divorcé, il la persuade de venir la rejoindre à New-York pour être près de ses enfants. Si Alison essaie pendant un temps de se conformer au désir de son nouveau compagnon, elle finit par lui échapper, signifiant qu'il est illusoire de vouloir capturer une vague. La fin de la deuxième saison nous montre qu'elle lui a imperceptiblement glissé entre les doigts pour retourner à la mer, parce qu'Alison est la mer, un élément qui nous était prédit dans les paroles du générique de début, composées pour la série par Fiona Apple :

I have only one thing to do and that's

To be the wave that I am and then

\section{Sink back into the ocean}

La différence entre l'affiche de promotion de la série et le générique donne à penser que l'image fixe représente la relation extra-conjugale du point de vue de Noah alors que le 
générique est une représentation visuelle du monde intérieur d'Alison. L'affiche est porteuse d'espoir alors que le générique est annonciateur du désastre à venir. Alors, Noah, dont le nom est fortement chargé culturellement, réussira-t-il à sauver son couple de la noyade ou est-ce que la puissance destructrice d'Alison finira par les submerger ? La suite au prochain épisode.

\section{NOTES}

1. Montauk is the device that drives the story forward. The environment of Montauk is the central character of our show because it becomes the framing device that the rest of the series takes place in. (Toutes les répliques ont été traduites par nos soins).

2. Fitzgerald, Francis Scott. The Great Gatsby. New York : Scribner, 1925.

3. Cole: This place smells like hell. [...] Do you smell that? You'd have to have a death wish to want to live here. [...] I feel like I'm gonna puke.

4. Arnoult, Pierre. «Le paysage dans tous ses états. Propos de géographes », Géoconfluences. 2004. Repéré àhttp://geoconfluences.ens.lyon.fr/doc/transv/paysage/PaysageScient.htm. Consulté le 15 février 2016.

5. Noah: I was a happy man back then. I was proud of my family. My first book had just come out. I'd... Everything I'd promised myself I'd achieve when I was a young man, I... - I'd done it. (E01S01)

6. "Everyone has one book in them. Almost nobody has two".

7. Noah: You don't think I could handle it?Alison: I think you're a summer person.

Noah: What's that mean?

Alison: Well, I think you have a fantasy of what life is out here. It's a small town. It's idyllic. We're simple, humble people who love the sea, right?

Noah: So what's it really like?

8. It's hard, though, you know. [...). You go out in the evening. You're not back till the morning. You sleep on the boat in shifts. It's cold. It's wet. The ocean is mean. (S01E03)

9. "I was a bit of a shipwreck fanatic when I was a child" (S01E04)

10. My mum? Where is she? I don't really know. She's like the wind. She goes where she wants. She does what she wants. (S01E04)

\section{AUTEUR}

\section{SANDRINE CHAPON}

Sandrine Chapon est Professeur à la faculté de droit de l'Université Grenoble Alpes depuis 2005 et docteure en anglais de spécialité depuis 2015. Dans le cadre de sa thèse, elle a suivi le tournage de la série "Trial", produite par Amazon (sortie prévue juin 2016) et interviewe plusieurs scénaristes de séries judiciaires (Leonard Dick, Jonathan Shapiro, Jason Tracey). Elle est l'auteure de 
plusieurs articles sur l'intérêt didactique des séries judiciaires pour l'apprentissage de l'anglais juridique. 